\title{
A special form of cerebral lacunae: expanding lacunae
}

Pascale Homeyer, Philippe Cornu, Lucette Lacomblez, Jacques Chiras, Christian Derouesné

\begin{abstract}
The case of a 42 year old man with headache, blurred vision, and diplopia allowed the description of a particular form of cerebral lacunae-that is, expanding lacunae. Brain MRI showed hydrocephalus and multiple lesions in the thalamomesencephalic region. The radiological features of these lesions were similar to the histological brain coronal section of a case reported in 1983 in which expanding lacunae were related to a dilatation of the perivascular spaces and a focal segmental necrotising angiitis. The role of the lymphatic drainage of the brain is discussed to explain the dilatation of the perivascular spaces. The hypothesis of a hydrodynamic factor being responsible for the expanding character of the lacunae was suggested by the location of the lesions and the influence of various clinical events on the symptomatology.
\end{abstract}

(F Neurol Neurosurg Psychiatry 1996;61:200-202)

Keywords: cerebral lacunae; hydrocephalus; lymphatic drainage of the brain; cerebral segmental angiitis

In 1983, Poirier et $a l^{1}$ described a 54 year old woman who developed a progressive subcortical dementia associated with multiple round hypodensities on CT in both thalami and the midbrain. Neuropathological examination showed that these hypodensities were due to dilatation of the perivascular spaces in the territory of the paramedian mesencephalothalamic artery. Expanding lacunae seem to be exceptional because we found only two previous case reports, with a similar histological structure but different anatomical locations. ${ }^{23}$ We had the opportunity to study a patient with MRI features very similar to the histological brain coronal section of the patient described by Poirier et al.

\section{Case report}

A 42 year old man was admitted to the neurosurgical department of the Hôpital de la Salpêtrière in June 1992 for headache associated with a 10 year history of blurred vision and diplopia. This patient had lived in
Madagascar for 17 years and had travelled to Venezuela and the West Indies. In 1985, he sustained a head injury with loss of consciousness of unknown duration followed by a two day post-traumatic amnesia. After the head trauma, the patient became aggressive and irritable and the visual disorders became more pronounced. He developed headache, scalp hyperaesthesia, and paraesthesia in the arms spreading to the fourth and fifth fingers of both hands.

On admission, he showed a Parinaud syndrome and a skew deviation. Fundus oculi were normal. Brain CT showed a low density subthalamic lesion, a moderate enlargement of the third ventricle, considerable dilatation of the lateral ventricles without periventricular lucency, and scattered calcifications near the grey-white matter junction in the cerebral cortex. Brain MRI showed multiple lesions involving the right part of the mesencephalon, extending into the right thalamic region (figure). These multiple lesions had the aspect of a bunch of grapes 2 to $15 \mathrm{~mm}$ diameter, separated by tissue which had the same density as the normal brain. The wall of the lateral ventricles seemed to be deformed by polycystic prints. These lesions presented a similar signal to CSF: highly hypointense on T1 weighted sequences and hyperintense on $\mathrm{T} 2$ weighted sequences. They were not enhanced by gadolinium. In view of the displacement of the posterior part of the third ventricle, the thinning of the sylvian aqueduct and of the quadrigeminal cistern, and the enlargement of the cerebral peduncles, these lesions have an expanding character; MRI excluded hind brain herniation and syringomyelia.

A parasitosis of the CNS was first considered. Serum antibodies suggested previous exposure to toxoplasma. Examination of CSF for cysticercosis (enzyme linked immunosorbent assay), hydatid cysts, and cryptococcus was negative. Serology for HIV was also negative. Despite the negativity of the serology for cysticercosis, a three week course of praziquantel was prescribed considering the several stays in endemic zones and the presence of cerebral calcifications. No clinical or radiological changes were noted during and after the treatment. A right ventriculoperitoneal shunt with a medium pressure Holter valve was inserted. The paraesthesia in the arms and headache 
MRI T1 weighted sequences. $(A)$ sagittal section; (B) coronal section.

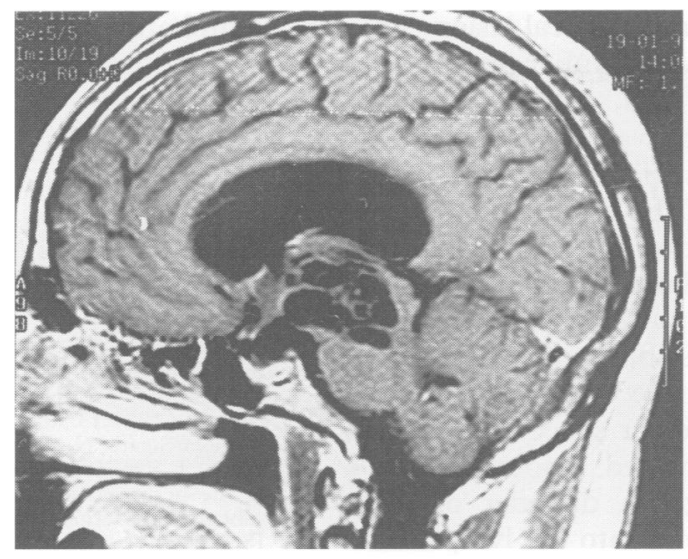

A

disappeared, and the visual symptoms improved. After a slight head trauma, the symptoms recurred immediately. Despite a shunt revision and a change of valve, a fluctuating diplopia persisted. No modification of the lesions was shown by MRI. In January 1993, headache and visual disturbances increased and temporarily became worse as a result of slight head injuries.

No abnormality was found in serum tests for systemic diseases. The CSF contained 0.64 $\mathrm{g} / \mathrm{l}$ of protein with a normal IgG ratio. A cerebral angiography was performed to exclude a cerebral vasculitis. The thalamic perforating arteries and geniculate arteries showed no parietal abnormalities but one of these arteries and one of the superior cerebellar arteries were displaced around the lacunae.

After iodine based contrast injections (given during the cerebral angiography and CT) and after a lumbar puncture, the patient's clinical state improved for two hours. A similar improvement was obtained by intravenous injections of hypertonic substances: dextran or glycerol. Treatment with $1 \mathrm{~g}$ daily acetazolamide for four days did not prolong the improvement after the lumbar puncture. Because of a possible segmental cerebral arteritis, the patient received $1 \mathrm{~g}$ methylprednisolone intravenously over three days, followed by $35 \mathrm{mg}$ daily of oral prednisone for six weeks. No change was seen.

\section{Discussion}

The aetiology of the lesions can only be hypothetical. This patient presented multiple cerebral lesions in the thalamomesencephalic region and hydrocephalus, so the first diagnosis considered was a parasitosis and, in particular, cerebral cysticercosis. The calcifications shown by CT were compatible with an old cysticercosis but there was no evidence of a recent cysticercosis: there were neither high density nodules within the cyst fluid, nor surrounding cerebral oedema as described in cerebral cysticercosis. ${ }^{4}$ The serology for cysticercosis was negative and treatment with praziquantel did not result in clinical or radiological improvement. The radiological appearance was very different from that of a cystic tumour such as cystic glioma or craniopharyngioma although a case of craniopharyngioma

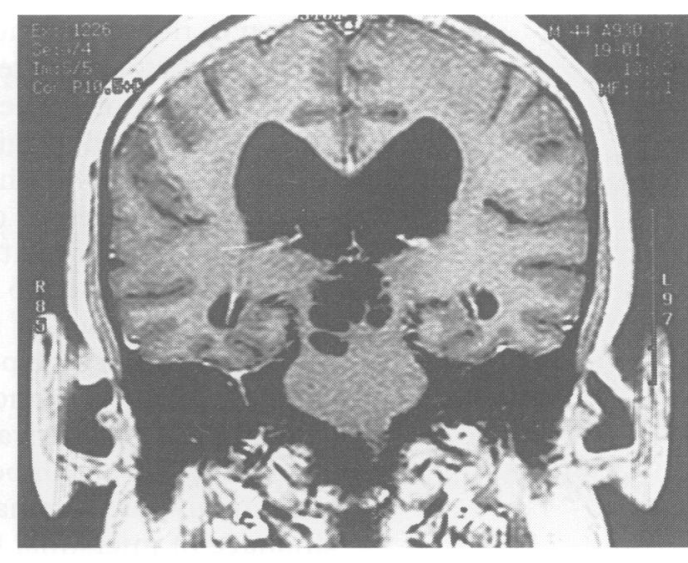

$B$

invading the thalamus, midbrain, and pons has been reported. ${ }^{5}$ We did not find in the medical literature any case of cerebral cysts with a histological examination of the cyst wall and with a topography and a radiological aspect that resembled those of our patient. The lesions did not have the intrasellar topography of a Rathke cleft cyst. Arachnoïd cysts are extraparenchymal and neuroepithelial cysts are not usually polycystic. Only one case of polycystic neuroepithelial cyst has been reported, in a 10 year old girl, but the histology was not studied. ${ }^{6}$ In our patient, the lesions had the topography of a vascular territory but, considering their radiological features, they were different from an old infarct. ${ }^{7}$ The features and the location of the lesions on the coronal sections of the MRI were similar to those from postmortem examination provided by Poirier et al. ${ }^{1}$

The histological structure of the lesions in the case of Poirier $e t a l^{1}$ was that of cerebral lacunae due to dilatation of the perivascular spaces or type III lacunae according to the classification of lacunae by Poirier and Derouesné. ${ }^{8}$ A permeable arteriole was situated in the centre of the cavities, which were lined by a single layer of pavement epithelium corresponding to the normal lining of Virchow-Robin spaces. Poirier et al found that these lacunae could compress or displace adjacent cerebral structures or bulge into the ventricles. ${ }^{1}$ The proximity of the ventricles and the presence of hydrocephalus was thought to explain the expanding character of the lesions by means of a hydrodynamic factor. In our patient, some clinical arguments support the role of a hydrodynamic factor: the fluctuations of the clinical features during the course of successive examinations, the deterioration after minor head injuries, the improvement of the symptoms after shunting and perfusion of hypertonic solutions, and the beneficial effect of the lumbar puncture. The improvement of the symptoms after perfusion of hypertonic solutions suggests that these lacunae are related to the breakdown of the blood-brain barrier, which could be responsible for the dilatation of the perivascular spaces. On the other hand, the improvement after the lowering of the CSF pressure suggests that their expanding nature is related to the CSF pressure. However, no clear difference could be 
shown in the size of the radiological lesions on the MRI, before or after the lumbar puncture.

In the case of Poirier et al, ${ }^{1}$ a localised segmental necrotising angiitis was found in a mesencephalic artery. This permeable arteriole situated in the centre of the cavities might be responsible for the dilatation of the perivascular spaces. In our case, no clinical, radiological, or biological evidence of a systemic arteritis was found. However, the possibility of a local segmental arteritis cannot be ruled out. The dilatation of the perivascular spaces might be due to poorly compensated impedance of interstitial fluid drainage. A disorder of the drainage of interstitial fluid into the lymphatic pathways has to be considered, although no techniques allow lymphatic drainage pathways from the human brain to be identified in vivo. These pathways are well described, however, in non-human mammals through tracer experiments. ${ }^{9} \mathrm{~A}$ major outflow pathway for extracellular fluid is the passage across arachnoïd villi to dural sinus blood. Another efflux pathway is the drainage along routes adjacent to cranial nerves (olfactory, optic, trigeminal, and acoustic nerves) and spinal nerves to regional lymph nodes. ${ }^{1011}$ In rats, the obstruction of the lymphatic drainage pathways of the brain by cervical lymphadenectomy results in ventricular dilatation in $80 \%$ of animals. ${ }^{12}$

In conclusion, we draw attention to this very special type of cerebral lacunae and to the possible role of a hydrodynamic factor and of the lymphatic drainage of the brain in its development.

We thank Professor Poirier for his expert opinion on this case and Miss Nancy Winchester for revising the English version of the manuscript.

1 Poirier J, Barbizet J, Gaston A, Meyrignac C. Démence thalamique. Lacunes expansives du territoire thalamomésencéphalique paramédian. Hydrocéphalie par sténose de l'aqueduc de Sylvius. Rev Neurol (Paris) 1983;139: 349-58.

2 Derouesné C, Gray F, Escourolle R, Castaigne P. Expanding cerebral lacunae in a hypertensive patient with normal pressure hydrocephalus. Neuropathol App Neurobiol 1987;13:309-20.

3 Benhaiem-Sigaux N, Gray F, Gherardi R, Roucayrol AM, Poirier J. Expanding cerebellar lacunae due to dilatation of the perivascular space associated with Binswanger's subcortical arteriosclerotic encephalopathy. Stroke 1987; 18:61087-92.

4 Davis LE, Kornfeld M. Neurocysticercosis: neurologic, pathogenic, diagnostic and therapeutic aspects. Eur pathogenic, diagnostic

5 Grover DW, Rorke LB. Invasive craniopharyngioma. $f$ Neurol Psychiatry 1968;31:580-2.

6 Nakasu Y, Handa J, Watanabe K. Progressive neurological deficits with benign intracerebral cysts. $\mathcal{F}$ Neurosurg 1986; 65:706-9.

7 Castaigne P, Lhermitte F, Buge A, Escourolle R, Hauw JJ, Lyon-Caen $O$. Paramedian thalamic and midbrain infarcts: clinical and neuropathological study. Ann Neurol 1981;10:127-48.

8 Poirier J, Derouesné C. Cerebral lacunae. A proposed new classification. Clin Neuropathol 1984;3:266.

9 Hunter JV, Batchelder KF, Lo EH, Wolf GL. Imaging techniques for in vivo quantification of extracranial lymtechniques for in vivo quantification of extracranial lym-
phatic drainage of the brain. Neuropathol Appl Neurobiol phatic drainage
$1995 ; 21: 185-8$.

10 Cserr HF, Knopf PM. Cervical lymphatics, the blood-brain barrier and the immunoreactivity of the brain: a new barrier and the immunoreactivity of
view. Immunol Today 1992;13:507-12.

11 Kida S, Weller RO, Zhang ET, Phillips MJ, Iannotti F. Anatomical pathways for lymphatic drainage of the brain and their pathological significance. Neuropathol Appl Neurobiol 1995;21:181-4.

12 Kida S. In: symposium on lymphatic drainage of the brain Neuropathol Appl Neurobiol 1995;21:173-4. 